\title{
Cognitive Model Selections in Co-existing Operation of Wireless Sensor Networks
}

\author{
Vasanth Iyer*, S. Sitharama Iyengar ${ }^{\dagger}$, Garmiela Rama Murthy*, \\ N. Parameswaran ${ }^{\llbracket, ~ V i r ~ P h o h a ~}{ }^{\ddagger}$ and Mandalika B. Srinivas ${ }^{\S}$ \\ *International Institute of Information Technology, Hyderabad, India - 500032 \\ ${ }^{\dagger}$ Louisiana State University, Baton Rouge, LA 70803, USA \\ ${ }^{\S}$ Brila Institute of Technology \& Science, Hyderabad Campus, Hyderabad-500078, India \\ ${ }^{\ddagger}$ Louisiana Tech University, Center for Secure Cyberspace, LA 70803, USA \\ TThe University of New South Wales, Sydney 2052, Australia \\ vasanth@research.iiit.ac.in;iyengar@csc.lsu.edu;phoha@coes.latech.edu;paramesh@csc.unsw.edu.au;srinivas@bits- \\ hyderabad.ac.in;rammurthy@iiit.ac.in
}

\begin{abstract}
In this paper, we describe the cognitive radios sharing the spectrum with licensed users and its effects on operational coexistence with unlicensed users. Due to the unlicensed spectrum band growing needs and usage by many IEEE 802.11 protocols, normal wireless radio operation sees high interference leading to high error rates on operational environments. We study the licensed bands and the characteristics of the unlicensed bands, as it is know that the licensed bands have a maximum limit of FCC interference for a licensed set of frequencies. The cognitive algorithm for this probabilistic model for the unlicensed users, uses a model which takes into account the threshold variable ratio $\frac{E_{b}}{N_{o}}$ and also calculates the lower-bound of the combined value of secondary user interference for overlapping frequencies with the primary user. By using simulation, we detect the primary user when the radio frequencies are known a priori and compare it when the frequencies are unknown and needs to cognitively detected. In our analysis we exploit the similarity measure seen at each sub-channel frequencies, which are due to multiple paths of the same reflected signal by maximizing the correlated information of the correlation matrix. For the general case the covariance matrix for blind source separation, we use ICA de-correlation methods and show that cognitive radios can efficiently identify users in complex situations.
\end{abstract}

Index Terms-Algorithm complexity, SDR and Cognitive Radios, Power Aware Radios, Wireless Sensor Network, Covariance Matrix, PCA, ICA.

\section{INTRODUCTION}

Cognitive radio and cognitive network studied here are both considered static. There are mobile primary users model for extensions to study specific signal estimation techniques. The cognition in their part has two common modes of interference avoidance. The first approach uses overlay to make up for the unused spectrum bandwidth and the second approach uses underlay in the form of interference control. The history of cognitive radio can be attributed to the thesis work of J. Mitola in 2000, where he coined "Cognitive Radio" for a form of radio that would change its performance by detecting its environment and changing accordingly. We like to find the tradeoffs between minimum spectrum power allocation and channel rate, when operating in overlapping frequencies with primary users.
We like to study the performance of deploying for Wireless Sensor Networks which uses the ISM band using IEEE 802.15.4 protocol in context of Cognitive networks. There has been a lot of emerging standards on inter-operability but none of them address the distributed nature of the spectrum. Some of the deployments have adapted to frequency reuse and orthogonal spectrum allocations to have least interference and better usage of the same spectrum. These implementations allow baseline reality and also taking into consideration of the non-linearity of the radios in practice which introduce errors during channel coding. We model

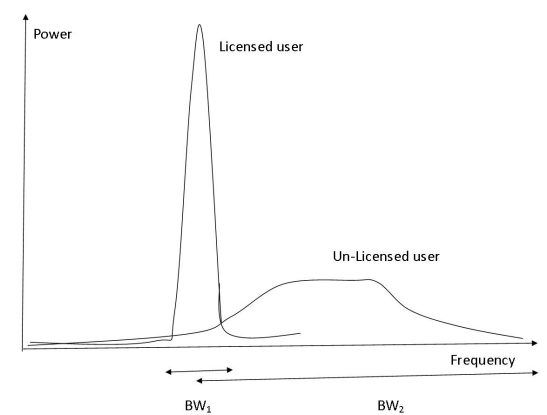

Fig. 1. Unlicensed user partially overlapping with primary user.

interference as unlicensed users partially overlapping with primary user as shown in Figure 2, which gives raise to co-channel interference. The varying parameters at the radio receivers are interference due number of overlapping channels and variation caused by mobility. The interference in mobility can be seen as the phase shift due to doppler and phase shift leading to delay spread due frequency. The two dimensional representation of interference varying with wireless range from the primary signal and the interfering signals is represented using covariance matrix. As we are interested in 
correlated channels with high signal to noise ratio (SNR), which leads to higher link quality and interference free reception during spectrum usage.

Rest of the paper is organized into: Section II describes related work in these emerging standards. In Section III, we outline architecture of the Cognitive Model using Interference avoidance (spectrum-overlay). In Section IV, interference versus energy model is described for a given bandwidth and channel capacity. In Section $\mathrm{V}$, the Rayleigh fading model is described in terms of mobility patters and how it can be represented as a twodimensional covariance matrix. Concluding section on simulated results describes the upper and lower-bound thresholds for maximizing covariance and ICA [8] for selecting coexisting channels for large Wireless Sensor Network.

\section{RELATED STANDARDS}

Due to the availability of Software Defined Radios and its ability to architect sophisticated spectrum sensing radios, the FCC in 2004 formed a working group to define 802.22 standards. The new standard was particularly to rural areas by sharing the television spectrum, the standard is expected to be completed by the first quarter of 2010 and with this some of the first networks could be deployed.

There are a number of elements that were set down for the basis of the 802.22 standard. These include items such as the system topology, system capacity and the projected coverage for the system. By setting these basic system parameters in place, the other areas fall into place. The parameters which effect such channel capacity, SNR, energy efficiency, BER and optimum radio modulation schemes for a given interference level to exist with other radios is described in Theorem 1 through Theorem 7.

\section{MODEL PREDICTION}

\section{A. Interference avoidance (spectrum overlay)}

Using the specification of 802.22, which relies on a central command controller, it would allow the base station to have large training samples collected from all the CPE's. The data-set can be used over time to predict the spectrum availability for cooperative future scheduling. The spectrum can be categorized into highly, medium and sparsely used. We expect the coverage in urban areas, where the network may be deployed would fall into the sparse spectrum category. The CPE's which are deployed remotely will collect the frequency of the detected signal and its time duration in its overlapping spectrum. The spectrum availability can be calculated with the overlapping intervals. Current radio design uses packed based count [2] and time-sampling [2] techniques. In the packed capture technique, the cognitive module becomes quite complex due to large number of packets and its demanding space requirements. In the other implementation Systematic Timer based Sampling
(STT), all the channels are sampled at an interval of $1 \mathrm{sec}-$ ond and provides an accurate measure of the spectrum activity to be recorded. The above techniques takes into account the capture of packets over the entire networks and uses cognitive network techniques.

In this paper, we discuss how to represent data-sets in two-dimensions which captures the interference model and the path-loss model for cognitive radio co-existence. These dynamic channel losses are used to de-correlate the signals of the primary and secondary users.

\section{B. IT Model (spectrum underlay)}

The concept of interference temperature [1] is identical to that of noise temperature. It is a measure of the power and bandwidth occupied by interference. Interference temperature $T_{I}$ is specified in Kevin and is defined as

$$
T_{I}\left(f_{c}, B\right)=\frac{P_{I}\left(f_{c}, B\right)}{k B}
$$

where $P_{I}\left(f_{c}, B\right)$ is the average interference power in Watts centered at $f_{c}$, covering bandwidth $B$ measured in Hertz. Boltzmann's [1] constant $k$ is $1: 38 \times 10^{-23}$.

\section{Ideal Model-Known Frequencies}

In the ideal interference temperature model we attempt to compute only the interference due to licensed signals. Assume our unlicensed transmitter is operating with average power $P$, and frequency $f_{c}$, with bandwidth $B$. Assuming that the radio knows the frequencies of the base station and the allowed bandwidth, then it needs to only filter frequencies in the range $f_{c}-B / 2+f_{c}+B / 2$ overlaps $n$ licensed signals, with respective frequencies and bandwidth of $f_{i}$ and $B_{i}$.

As shown in Figure 1, we need to guarantee that

$$
T_{I}\left(f_{i}, B_{i}\right)+\frac{M_{i} P}{k B_{i}} \leq T_{L} f_{i}, 1 \leq i \leq n
$$

Note the introduction of constants $M_{i}$. This is a fractional value between 0 and 10 as shown in Table IV, representing a multiplicative attenuation due to fading and path loss between the unlicensed transmitter and the licensed receiver. Section II described the 802.11 standard, which allows to define the licensed user. The model co-existing needs only to know which are the overlapping frequencies other than the given standard specification of frequency and bandwidth. The second step is to measure $T_{I}$ in the presence of the licensed signal. Assuming we know the signal characteristics and the wireless losses we can use correlation of the measured interference, which will help isolate the redundant signal interference. Also, if we have precise knowledge of the signal's bandwidth $B$ and center frequency $f_{c}$, we can approximate the interference temperature

$$
T_{I}\left(f_{c}, B\right) \approx \frac{P\left(f_{c}-B / 2-\tau\right)+P\left(f_{c}+B / 2+\tau\right)}{2 k B}
$$

where $P(f)$ is the sensed signal power at frequency $f$ and $\tau$ is a safety margin of a few $\mathrm{kHz}$. 


\section{Generalized Model-Unknown Frequencies}

In cases where there is no prior knowledge, which could be a new network environment then, we need to apply interference temperature model to the entire frequency range of operation to detect any primary user. This is typically the case with blind source separation.

$$
T_{I}\left(f_{c}, B\right)+\frac{M P}{k B} \leq T_{L} f_{c}
$$

Assuming each licensed signal has power $P_{i}$ and otherwise the interference floor is defined by the thermal noise temperature $T_{N}$, we can transform (4) into the following: $K B T_{L}\left(f_{c}\left(B-B_{i}\right)+k B T_{N} \Sigma_{j=1}^{n} B_{j} \leq \sum_{j=1}^{n} B_{j} P_{j} \forall 1 \leq i \leq n\right.$

In a simple case with only one licensed receiver, the inequality simplifies to

$$
\frac{K B T_{L}}{P_{1}-k B T_{N}} \leq \frac{B_{1}}{B-B_{1}}
$$

Latter in the analysis we show how to measure and decorrelate in such complex environments.

\section{Modeling Mobility in Wireless Channels}

Rayleigh fading is used to describe the characteristic of the wireless channels, which are used by wireless receivers. The Rayleigh model assumes that signal has passed through such a medium and will vary randomly or fade according to Rayleigh model. The Doppler power spectral density of a fading channel describes how much spectral broadening it causes. The effect on pure signal, when it passes through such a channel.

$$
S_{\nu}=\frac{1}{\pi f_{d} \sqrt{1-\left(\frac{\nu}{f_{d}}\right)^{2}}}
$$

Where $\nu$ is the frequency shift relative to the carrier frequency. The equation is valid only for values of $\nu$ between $\pm f_{d}$. The Doppler model as shown in equation (15) and the Rayleigh model described here and in equation (24), we can extend it to simulate mobility by summing up the sinusoidal. The calculation of the coefficient of the real and imaginary parts used by the Rayleigh model can be redefined for a scatter, which is uniformly distributed around a circle at angles $\alpha_{n}$ with $k$ rays emerging from each scatter.

In this model we use multiple radio receivers $R_{p}, R_{s 1}, R_{s 2} \ldots R_{s m}$. The normalized autocorrelation function of a Rayleigh faded channel with motion at a constant velocity is a zeroth-order Bessel function of the first kind:

$$
R \tau=J_{0}\left(2 \pi f_{d} \tau\right)
$$

\section{A. Level Crossing Rate}

The level crossing rate is a measure of the rapidity of the fading. It quantifies how often the fading crosses some threshold, usually in the positive-going direction. For Rayleigh fading, the level crossing rate is:

$$
L C R=2 \pi f_{d} \rho \exp ^{-\rho^{2}}
$$

where $f_{d}$ is the maximum Doppler shift and $\rho$ is the threshold level normalized to the root mean square (RMS) signal level:

$$
\rho=\frac{R_{\text {thresh }}}{R_{\text {rms }}}
$$

Let us design a way to detect and isolate the primary user $R_{p}$ and a secondary users $R_{s 1}, R_{s 2} \ldots R_{s m}$ for channels $c h_{1}, c h_{2}, c h_{3} \ldots c h_{48}$. For single mobile receivers the interference noise due to path-loss has a single spike, but for multiple users the interference noise due to pathloss degrades into a Gaussian curve [5]. From equation (2), we calculate a values $M_{i}$ for the interference seen at multiple channels in time.

$$
M_{\text {cov }}=\left(\begin{array}{ll}
\rho_{11} & \rho_{12} \\
\rho_{21} & \rho_{22}
\end{array}\right)
$$

To maximize the correlation we need to optimally select the diagonal elements of the matrix shown in equation (11) and values in Table IV, such that the equation below (12) can be used to select channels which can differentiate primary user from the secondary user's interference.

$$
G=\max \operatorname{det}(M)=\rho_{11}=\rho_{22}=1
$$

\section{Simulation}

TABLE II

Cognitive Channel Interference Simulation SetuP

\begin{tabular}{|rrr|}
\hline Methods & Model & Metric \\
\hline Mobility Traces & OMNET++ & Doppler \\
Propagation & Rayleigh-Jake's & Phase, freq \\
Radios & 1 Primary user & $T_{I}$ Cognitive \\
Channel & 48 Sub-channels & $M_{i}$ \\
Noise & 1 Secondary user & Floor-noise \\
Covariance & R-System & $\rho_{\text {cov }}$ \\
ICA & R-System & $\rho_{\text {uncov }}$ \\
\hline
\end{tabular}

IT model described in equation (2), we calculate different values for the variable terms $T_{I}\left(f_{i}, B_{i}\right)$ and $\frac{M_{i} P}{k B_{i}}$. A OMNET mobility framework simulator, uses interference modeling as derived in Section IV and Table II. We use two models to analyze the data collected from the mobility simulator, one uses the covariance technique $[4,6]$ to optimize as shown in equation (12), when there is not too much of co-channel interference and the seconds methods uses ICA, which de-correlates under the presence of heavy co-channel interference.

\section{A. Two Dimensional Representation of Interference using Covariance}

The Figure 2 shows different values of $M_{i}$ seen at the primary receiver with no secondary users, as it can 


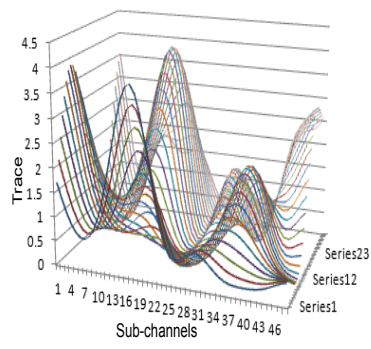

Fig. 2. Ideal Model - Primary user with mobility in time.

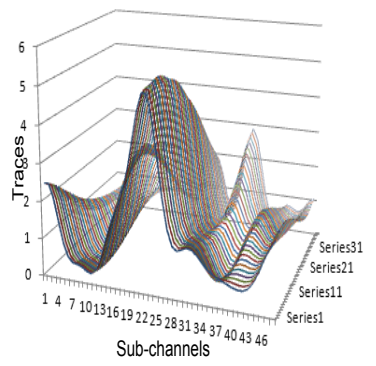

Fig. 3. General Model Primary user with coexisting Secondary user in time.

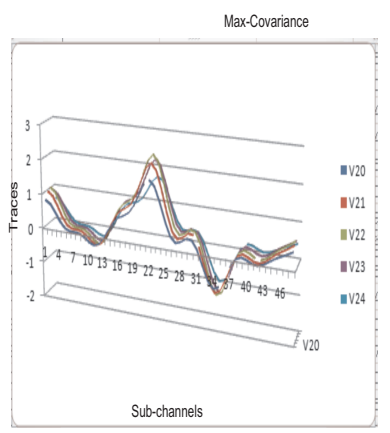

Fig. 4. Normalized $\rho_{\text {cor }}$ coefficient of the optimal correlated channels.



Fig. 5. Normalized $\rho_{\text {uncor }}$ co-efficient of uncorrelated channels.

TABLE I

VALUES OF THE DIAGONAL ELEMENTS FROM TABLE IV. (VALUES COMPARED WITH ICA-METHOD)

\begin{tabular}{|c|c|c|c|c|c|c|}
\hline Values of $\rho_{i, j}$ from Table IV & Measured $M_{H i}$ & Measured $M_{\text {low }}$ & Ideal Upper-Bound & Ideal Lower-Bound & General Upper Bound & General Lower Bound \\
\hline$\rho_{\text {cor }}=1.313362745$ & 3.792 & 0.08 & 0.46 & 0.32 & 1.41028417 & 1.22920039 \\
$\rho_{\text {cor }}=1.3747417$ & 4.362 & 0.022 & 1.821411 & 0.345488 & 2.0216711 & -1.22920039 \\
$\rho_{\text {cor }}=2.07415429$ & 4.222 & 0.034 & 2.235424 & 0.415796 & 2.0216711 & 0.8926338 \\
$\rho_{\text {cor }}=2.086886414$ & 3.917 & 0.063 & 2.229352 & 0.530349 & 2.3109796 & 0.21394222 \\
$\rho_{\text {cor }}=2.420906219$ & 4.073 & 0.047 & 2.420906 & 0.09 & 2.7578501 & -0.74375612 \\
\hline$\rho_{\text {uncor }}=0.816974583$ & 4.362 & 0.013 & 1.399701 & 0.320941 & 1.30032658 & 0.03084368 \\
\hline
\end{tabular}

be seen that it has unique peaks, which vary in time. The measured co-efficient are shown in columns of Table IV and the corresponding covariances are calculated in calculated columns of the current table. Initially, when only the primary user is using the spectrum and has mobility with constant fading and changing wireless range, the signals seen at the receiver has a sharp spike which is shown in Figure 2. The corresponding Figure 3 shows the effects of interference at the primary receiver, the plot does not have any spatial or time varying properties, as it is uniformly distributed, which follows a Gaussian distribution. To separate the interference from the secondary users, we need to compute the lower and upper bounds of the interference floor, which is computed by the thermal noise temperature $T_{N}$. The coefficient of the covariance matrix of all the 5 data-sets are chosen to maximize $G$ as shown in Table IV. As we to use the correlation between signals, which are due to multi-path scattering, we plot the upper bound response of the attenuation of the channels, this is shown in Figure 4 , which are seen completely correlated and described in equation (3).

\section{B. Estimation of Interference using ICA}

The above method uses correlation matrix to maximize the determinant to find the primary user and secondary users. ICA uses a method which seeks components, which are varying independently and thus differentiates the primary user and the rest. This methods is preferred when the noise level is very high in the channels. The measured coefficients for ICA analysis using
R-System package fastICA [3], are tabulated in Table $\operatorname{IV}\left(I C A_{1}, I C A_{2}\right.$, where users=2), $\rho_{\text {uncorr }}=1.30032658$ for number of signal equal to two, which is approximately lower bound of the last result set $\rho_{\text {corr }}=$ 1.313362745. Figure 5 shows how ICA can perform well when the sources are unknown and blind or shadowed to measurements, when the noise level is above the given interference threshold, as shown in equation (6).

\section{CONCLUSIONS}

In the power-aware signal analysis we compare the ideal spectrum model with the generalized spectrum model to obtain obtain upper and lower bounds of the thermal interference for the variable frequency range of primary user. We improvise by calculating the floor noise due to co-channel interference and detecting the primary user with least power as in the case of 802.22 standard. To find the performance of our method, when the primary user frequencies are unknown which is the case in wireless sensor networks, we compute ICA for the entire spectrum and show that the primary user detection is possible when coexisting with secondary users or when noise dominates the desired feature thresholds.

\section{ACKNOWLEDGEMENTS}

Authors like to thank Dr. Dietmar Janetzko in introducing R-System, which enabled standard comparison of the results discussed in the simulations. 


\section{INTERFERENCE VS ENERGY MODEL}

\section{Theorem 1 - Co-channel Interference}

$$
S I R=10 \log _{10}\left[\left(\frac{D}{R}\right)^{n} K_{l}^{-l}\right]
$$

Gaussian Model

- Adjacent Channel Interference

$$
S I R=C \frac{P_{T X}}{d^{n}}\left(\frac{P_{T X}^{\prime} \int_{w} G(f) d f}{(d)^{n}}\right)^{-1}
$$

Transmitter sensitive

- Multi-path fading

$$
\cos (2 \Pi f t+\theta) \Rightarrow \text { Doppler Spectrum Model }
$$

The effects of fading $\theta$ can be combated by using diversity to transmit the signal over multiple channels that experience independent fading and coherently combining them at the receiver. The probability of experiencing a fade in this composite channel is then proportional to the probability that all the component channels simultaneously experience a fade, a much more unlikely event.

As the IT model described in equation (2), we calculate different values for the variable terms $T_{I}\left(f_{i}, B_{i}\right)$ and $\frac{M_{i} P}{k B_{i}}$. The reliability of a digital system is measured in terms of the error rate in the transmission link. BER - Bit Error Rate, SER - Symbol Error Rate, FER - Frame Error Rate, PER - Packet Error Rate. The SER characterizes the performance of the modulator. The BER is measured at the bit-level in terms of the number of bits that are received erroneously.

\section{A. Channel Capacity}

Theorem 2 Shannon showed that in an AWGN channel, the maximum bit-rate $C$ that can be achieved with arbitrarily low error rate over a given transmission bandwidth $W T$ is bounded by the expression below:

$$
\begin{gathered}
E_{b}=\frac{E_{s}}{\log _{2} M} \\
\bar{E}_{b}=\sum \frac{p_{i} E_{s}^{i}}{\log _{2} M}
\end{gathered}
$$

where $p_{i}$ is the probability for the occurrence of the $i^{\text {th }}$ symbol with energy.

$$
E_{b}=\frac{\max E_{s}^{i}}{\log _{2} M}
$$

Theorem 3 Where $E_{b}$ is expressed in terms of the peak symbol energy in the signal constellation. $E_{s}^{i}$. where $\gamma=E_{b}$ and $\bar{\gamma}=\bar{E}_{b}$

$$
\frac{C}{W_{T}} \leq \log _{2}\left(1+\frac{P}{N_{o} W_{T}}\right)=\log _{2}\left(1+\bar{\gamma} \frac{C}{W_{T}}\right)
$$

Symbol Energy:

$$
E_{b}=\frac{E}{\log _{2} M}
$$

where $P / N_{o} W_{r}$ is the $S N R, C / W_{T}$ is the maximum achievable bandwidth efficiency in bps/Hz, and $\gamma$ is the average $E_{o} / N_{o}$ defined by equation (2).

\section{B. Error Rate Bounds.}

Theorem 4 SER on the otherhand is measured at the symbol level in terms of the number of symbols that are in error. A symbol error is made when the received signal falls outside of its decision region. A symbol error leads to a bit errors as the symbol is erroneously mapped to an incorrect bit-pattern. Let $n$ be the number of bits per symbol. Then, SER may be bounded in terms of BER as shown below:

$$
P_{b} \leq P_{s} \leq n \cdot P_{s}
$$

To express PER in terms of BER

$$
P_{p}=1-\left(1-P_{b}^{L_{p}}\right)
$$

\section{A basic measure using BER}

Theorem 5 SER, FER, and PER all depend on BER, a basic measure for digital system is based on BER which can be expressed in terms of $\frac{E_{b}}{N_{o}}$, where $E_{b}$ is the energy per bit and $N_{o}$ is the equivalent noise spectral density over the signal bandwidth. The variations of BER with $\frac{E_{b}}{N}$ depends on the channel and the type of the demodulator. Channel Models:(see Table III for BPSK-modulation)

- $A W G N$

$$
P_{b}=Q\left(\sqrt{\frac{2 E_{b}}{N_{o}}}\right)
$$

- Rayleigh Fading

$$
P_{b}=\frac{1}{2}\left(1-\frac{\sqrt{E_{b} / N_{o}}}{1+E_{b} / N_{o}}\right) \approx \frac{1}{4 E_{b} / N_{o}}
$$

D. Design goals for using BER

Theorem 6 Additive white Gaussian noise (AWGN) in general, in an channel, BER is exponentially related to $E_{b} / N_{o}$, while in a fading channel, BER is inversely related to $E_{b} / N_{o}$.

For a given BER, a digital system with lower $\frac{E_{b}}{N_{o}}$ requires lower transmission power, which can improve battery lifetime of the communication device and the system capacity.

\section{E. Energy Efficiency}

Theorem 7 Energy efficiency can be more accurately defined, when taking into account both energy and bandwidth, we define $f(x)$ of a system to be the amount of $E_{b} / N_{o}$ required for a given bandwidth efficiency:

\section{REFERENCES}

[1] T. Charles Clancy, and William A. Arbaugh. Measuring Interference Temperature, Internal Report, Department of Computer Science, University of Maryland, College Park. Laboratory for Telecommunication Sciences, Department of Defense. 
TABLE III

ENERGY EFFICIENCY COMPARISON

\begin{tabular}{|c|c|c|c|}
\hline BPSK & 9.09 & 1 & 11.0 \\
GMSK & 10.8 & 1.35 & 11.5 \\
QPSK & 9.09 & 2 & 16.5 \\
8-PSK & 19.82 & 3 & 11.8 \\
16-PSK & 55.41 & 4 & 6.8 \\
32-PSK & 171.2 & 5 & 3.6 \\
8-QAM & 13.93 & 3 & 16.8 \\
BFSK & 17.78 & 1 & 5.6 \\
\hline
\end{tabular}

TABLE IV

Covariance Matrix of Wireless Traces and Corresponding Sub-Channel Frequencies

\begin{tabular}{|c|c|c|c|c|c|c|c|c|c|c|c|c|}
\hline Range & $\rho_{20}$ & $\rho_{21}$ & $\rho_{22}$ & $\rho_{23}$ & $\rho_{24}$ & $M_{20}$ & $M_{21}$ & $M_{22}$ & $M_{23}$ & $M_{24}$ & $I C A_{1}$ & $I C A_{2}$ \\
\hline Trace1 & 0.819005 & 1.009957 & 1.065732 & 0.934069 & 0.652786 & 3.923 & 4.65 & 5.102 & 5.059 & 4.484 & 1.208655 & -1.62622 \\
\hline Trace2 & 0.719087 & 0.886273 & 0.93748 & 0.825654 & 0.582044 & 3.917 & 4.647 & 5.133 & 5.142 & 4.609 & 0.982308 & -1.33105 \\
\hline Trace3 & 0.497145 & 0.612625 & 0.648628 & 0.572306 & 0.404752 & 3.9 & 4.628 & 5.148 & 5.208 & 4.722 & 0.523269 & -0.90737 \\
\hline Trace4 & 0.25082 & 0.30958 & 0.325421 & 0.282977 & 0.194931 & 3.871 & 4.595 & 5.145 & 5.258 & 4.821 & 0.042144 & -0.58777 \\
\hline Trace5 & 0.078649 & 0.098259 & 0.09747 & 0.074467 & 0.038239 & 3.832 & 4.546 & 5.126 & 5.291 & 4.906 & -0.27233 & -0.48044 \\
\hline Trace6 & 0.01421 & 0.019515 & 0.010436 & -0.00869 & -0.0285 & 3.782 & 4.484 & 5.089 & 5.307 & 4.976 & -0.37232 & -0.5348 \\
\hline Trace7 & 0.012564 & 0.017693 & 0.006658 & -0.01512 & -0.03688 & 3.722 & 4.408 & 5.036 & 5.305 & 5.031 & -0.36001 & -0.61612 \\
\hline Trace8 & -0.00621 & -0.00555 & -0.01866 & -0.03849 & -0.05462 & 3.652 & 4.319 & 4.967 & 5.287 & 5.07 & -0.39222 & -0.61694 \\
\hline Trace9 & -0.08871 & -0.10729 & -0.1273 & -0.13572 & -0.12502 & 3.572 & 4.218 & 4.883 & 5.252 & 5.094 & -0.55236 & -0.5168 \\
\hline Trace10 & -0.206 & -0.25179 & -0.28119 & -0.27294 & -0.22383 & 3.484 & 4.105 & 4.785 & 5.2 & 5.101 & -0.7834 & -0.35516 \\
\hline Trace11 & -0.27255 & -0.33404 & -0.36642 & -0.34501 & -0.27091 & 3.388 & 3.983 & 4.673 & 5.133 & 5.092 & -0.93449 & -0.15592 \\
\hline Trace12 & -0.20931 & -0.25737 & -0.27685 & -0.25164 & $\begin{array}{r}-0.18709 \\
\end{array}$ & 3.284 & 3.851 & 4.549 & 5.05 & 5.068 & -0.87724 & 0.11665 \\
\hline Trace13 & -0.00643 & -0.01008 & 0.001782 & 0.023084 & 0.043099 & 3.173 & 3.71 & 4.413 & 4.953 & 5.028 & -0.60744 & 0.529308 \\
\hline Trace14 & 0.265026 & 0.320577 & 0.374369 & 0.39068 & 0.351435 & 3.057 & 3.563 & 4.266 & 4.843 & 4.973 & -0.24663 & 1.081212 \\
\hline Trace15 & 0.501131 & 0.607728 & 0.699525 & 0.714221 & 0.625923 & 2.935 & 3.409 & 4.111 & 4.719 & 4.903 & 0.053843 & 1.631356 \\
\hline Trace16 & 0.642563 & 0.779736 & 0.893462 & 0.905977 & 0.787305 & 2.81 & 3.251 & 3.947 & 4.585 & 4.82 & 0.240909 & 1.92233 \\
\hline Trace17 & 0.719088 & 0.874153 & 0.9919 & 0.990327 & 0.843886 & 2.681 & 3.089 & 3.777 & 4.439 & 4.723 & 0.409605 & 1.719841 \\
\hline Trace18 & 0.826483 & 1.008641 & $\begin{array}{l}1.122246 \\
\end{array}$ & 1.084467 & $\begin{array}{l}0.0884460 \\
0.88469\end{array}$ & $\begin{array}{l}2.001 \\
2.549\end{array}$ & 2.924 & 3.602 & $\begin{array}{l}4.459) \\
4.284\end{array}$ & 4.614 & 0.730731 & 0.987333 \\
\hline Trace19 & 1.047695 & 1.283933 & 1.399701 & 1.304821 & 1.009793 & 2.416 & 2.758 & 3.422 & 4.121 & 4.493 & 1.300327 & -0.03084 \\
\hline Trace20 & $1.37 \overrightarrow{4} 742$ & 1.688408 & 1.821411 & 1.66533 & 1.250253 & 2.282 & 2.592 & 3.24 & 3.951 & 4.362 & 2.021671 & -0.89263 \\
\hline Trace21 & 1.688408 & $2.07 \overrightarrow{4} 154$ & 2.235424 & 2.040148 & 1.527146 & 2.148 & 2.427 & 3.056 & 3.775 & 4.222 & 2.612485 & -1.18124 \\
\hline Trace22 & 1.821411 & 2.235424 & $2.42 \overrightarrow{0} 906$ & 2.229352 & 1.692795 & 2.015 & 2.264 & 2.872 & 3.595 & 4.073 & 2.75785 & -0.74376 \\
\hline Trace23 & 1.66533 & 2.040148 & 2.229352 & $2.08 \overrightarrow{6} 886$ & 1.625188 & 1.884 & 2.103 & 2.689 & 3.412 & 3.917 & 2.31098 & 0.213942 \\
\hline Trace24 & 1.250253 & 1.527146 & 1.692795 & 1.625188 & $1.31 \overrightarrow{3} 363$ & 1.755 & 1.947 & 2.508 & 3.227 & 3.754 & 1.410284 & 1.2292 \\
\hline Trace25 & 0.741164 & 0.900631 & 1.023418 & 1.024321 & 0.875703 & 1.63 & 1.795 & 2.33 & 3.041 & 3.587 & 0.425831 & 1.834546 \\
\hline Trace26 & 0.345488 & 0.415796 & 0.494383 & 0.530349 & 0.492484 & $\begin{array}{l}1.508 \\
1.508\end{array}$ & 1.649 & 2.157 & 2.855 & 3.416 & $\begin{array}{l}-0.24559 \\
-0\end{array}$ & 1.806442 \\
\hline Trace27 & 0.190847 & 0.228611 & 0.278069 & 0.307936 & 0.295826 & 1.39 & 1.509 & 1.988 & 2.671 & 3.243 & -0.40598 & 1.252722 \\
\hline Trace28 & 0.249278 & 0.303375 & 0.342897 & 0.340112 & 0.287338 & 1.278 & 1.375 & 1.825 & 2.49 & 3.068 & -0.16516 & 0.503049 \\
\hline Trace29 & 0.361162 & 0.443031 & 0.481365 & 0.445902 & 0.341709 & 1.171 & 1.249 & 1.669 & 2.313 & 2.892 & 0.142369 & -0.11559 \\
\hline Trace30 & 0.340436 & 0.418967 & 0.447248 & 0.400879 & 0.29129 & 1.069 & 1.13 & 1.521 & 2.14 & 2.718 & 0.173599 & -0.47131 \\
\hline Trace31 & 0.096809 & 0.120941 & 0.118359 & 0.087717 & 0.041238 & 0.974 & 1.019 & 1.38 & 1.972 & 2.545 & -0.20864 & -0.6557 \\
\hline Trace32 & -0.30748 & -0.37391 & -0.42541 & -0.42628 & -0.36486 & 0.884 & 0.917 & 1.247 & 1.811 & 2.375 & -0.86257 & -0.85629 \\
\hline Trace33 & -0.69662 & -0.84961 & -0.95089 & -0.9277 & -0.76653 & 0.801 & 0.822 & 1.123 & 1.657 & 2.208 & -1.46869 & -1.17292 \\
\hline Trace34 & -0.89114 & -1.0866 & -1.21674 & -1.18823 & -0.98316 & 0.725 & 0.736 & 1.008 & 1.51 & 2.046 & -1.7374 & -1.51336 \\
\hline Trace35 & -0.81468 & -0.99227 & -1.11691 & -1.10031 & -0.92121 & 0.655 & 0.658 & 0.902 & 1.371 & 1.89 & -1.58168 & -1.64597 \\
\hline & & -0.64646 & -0.73394 & -0.73324 & -0.6253 & 0.591 & 0.587 & 0.805 & 1.241 & 1.739 & -1.14928 & -1.37084 \\
\hline Trace37 & $\begin{array}{l}-0.3080 \\
-0.19892\end{array}$ & $\begin{array}{l}-0.040+0 \\
-0.24155\end{array}$ & -0.27719 & $\begin{array}{l}-0.75324 \\
-0.28155\end{array}$ & $\begin{array}{r}-0.0235 \\
-0.24515\end{array}$ & 0.533 & $\begin{array}{l}0.525 \\
0.525\end{array}$ & 0.717 & $\begin{array}{l}1.241 \\
1.119\end{array}$ & $\begin{array}{l}1.7595 \\
1.595\end{array}$ & $\begin{array}{r}-1.14920 \\
-0.7112\end{array}$ & -0.674 \\
\hline
\end{tabular}

[2] T. Bheemarjuna Reddy, B.S. Manoj, and Ramesh Rao, Time-Based Sampling Strategies for Multi-channel Wireless Traffic characterization in Tactical Cognitive Networks, in Proc. of IEEE MILCOM 2008, November 2008.

[3] Owen Jones, Robert Maillardet, and Andrew Robinson. Introduction to Scientific Programming and Simulation Using R. Chapman \& Hall/CRC, Boca Raton, FL, 2009. ISBN 978-1-4200-6872-6.

[4] P.T. Krishna Kumar, S.S Iyengar, Vir Phoha. Classification of radio elements using mutual information: A tool for geological mapping, Int. J. Appl. Earth Observ. Geoinform. (2008), doi:10.1016/j.jag.2007.12.001

[5] Bristow, Q., 1983. Airborne g-ray spectrometry in uranium exploration,principles and current practice. Int. J. Appl. Radiat. Isot. 1,199229 .

[6] Vasanth Iyer, G.Rama Murthy and M.B. Srinivas. Training Data Compression Algorithms and Reliability in Large Wireless Sensor Networks. International Journal On Smart Sensing and Intelligent Systems, Vol. 1, No. 4, pp 480 485, December 2008.

[7] Vasanth Iyer, G.Rama Murthy and M.B. Srinivas. Training Data Compression Algorithms and Reliability in Large Wireless Sensor Networks. International Journal On Smart Sensing and Intelligent Systems, Vol. 1, No. 4, pp 480 485, December 2008.

[8] Richard O. Duda, Peter E. Hart, David G. Stork, Pattern Classification, Wiley Interscience, ISBN: 0471056693 Edition 2nd, 2008. 